\title{
Automated Brain Tumor Segmentation on MR Images Based on Neutrosophic Set Approach
}

\author{
Mohan J \\ Department of Electronics and Communication Engg., \\ Vignan University, Vadlamudi, \\ Gundur Dt, Andrapradesh, India-522213. \\ jaimohan12@gmail.com
}

\author{
Krishnaveni V \\ Department of Electronics and Communication Engg., \\ P S G College Technology \\ Coimbatore, Tamilnadu, India-641004. \\ vk@ece.psgtech.ac.in
}

School of Science, Technology and Engineering Management, Saint Thomas University, Miami Gardens

FL 33054, USA.

yguo@stu.edu

\begin{abstract}
Brain tumor segmentation for MR images is a difficult and challenging task due to variation in type, size, location and shape of tumors. This paper presents an efficient and fully automatic brain tumor segmentation technique. This proposed technique includes non local preprocessing, fuzzy intensification to enhance the quality of the MR images, $k$ - means clustering method for brain tumor segmentation. The results are evaluated based on accuracy, sensitivity, specificity, false positive rate, false negative rate, Jaccard similarity metric and Dice coefficient. The preliminary results show $100 \%$ detection rate in all 20 test sets.
\end{abstract}

Keywords- Brain Tumor, k-means clustering, Magnetic Resonance Imaging, Neutrosophic Set, Wiener.

\section{Introduction}

Magnetic resonance imaging (MRI) is widely used to visualize brain structures such as white matter, grey matter and ventricles, and to detect abnormalities in the brain. In general, different MRI sequences are acquired for better visualization of abnormalities with different contrast properties. Brain tumor segmentation in MRI is an important step in medical diagnosis because it provides information associated to anatomical structures as well as potential abnormal tissues necessary to treatment planning and patient follow-up [1]. Typically, radiologists perform manual tumor segmentation and impart the details to neurologists. This is a timeconsuming process due to huge volume of images. In addition, the performance of manual segmentation is affected by the radiologists' fatigue. Therefore, computer aided brain tumor segmentation from MRI data has been an active and promising research area in recent years. However, it is still a challenging problem to obtain the accurate and reproducible segmentation and characterization of tumors automatically due to the high variation in appearance of tumor tissues among different patients.
Most of the existing techniques for brain tumor segmentation are not fully automatic due to expert-required model training or user-guided initialization or other user interaction, which leave significant space for increased automation, applicability and accuracy. The development of computer aided diagnosis (CAD) systems for brain tumor segmentation involves automatically assessing the probability of abnormality, and then highlighting the potentially abnormal regions for radiologists for further inspection. As a result, the probability of overlooking the tumor and the effort of manual examination of the whole 3D image are reduced. For this purpose, the CAD system should have the capability of fully automated segmentation of tumors.

The existing work on automatic brain tumor segmentation methods are broadly classified into region-based and contour based methods. In the region-based methods, Clark et al proposed an automatic tumor segmentation from MRI using knowledge- based techniques with multispectral analysis [2]. First, initial segmentation is performed by an unsupervised clustering algorithm. The segmented image, along with cluster centers for each class is provided to a rule based expert system which extracts the intracranial region. Multispectral histogram analysis separates suspected tumor from the rest of the intracranial region, with region analysis used in performing final tumor labeling. Kaus et al proposed a method for automatic segmentation of small brain tumors using a statistical classification method and atlas registration [3].

Moon et al have used the expectation maximization (EM) algorithm and atlas prior information [4]. Prastawa et al proposed a brain tumor segmentation frame work based on EM algorithm and outlier detection, where tumors are considered as outliers of the Gaussian model. These techniques detect tumor regions using a registered brain atlas as a model for healthy brains [5]. Except for the professional work of atlas generation, large deformation of tumors requires significant modification of the atlas which may lead to poor results. Much more flexible techniques without priori information were developed to overcome these problems. 
Philips et al first applied the fuzzy C-means clustering to segment brain tumors [6]. One of its drawbacks is that human interaction is required. Liu et al adapted the fuzzy connectedness framework for tumor segmentation with limited user interaction [7]. The fuzzy connectedness method has been applied by Moonis et al [8]. In this semi-automatic method, the user must select the region of the tumor. The calculation of the connectedness is achieved in this region and the tumor is delineated in $3 \mathrm{D}$ as a fuzzy connected object containing the seed points selected by the user. Dou et al proposed a fuzzy information fusion framework for brain tumor segmentation [9]. This method is sensitive to noise and needs some user interactions. Fully automated brain tumor segmentation from MR images based on improved fuzzy connectedness algorithm was proposed by Harati et al [10].However, the fuzzy model works well only for hyper intensity (fully enhanced) tumors and exhibit poor performance on detecting non-enhanced tumors.

In contour based methods, Lefohn et al applied level set implementation for brain tumor detection but for initialization of a first segmentation, in which user interaction is required [11]. Based on the visual inspection of the results of first segmentation, the level set parameters are tuned and the segmentation process is repeated. Ho et al incorporated region competition into level-set algorithm for brain tumor segmentation to overcome the initialization and weak edge leakage problems [12]. This method has high computational cost. Contour-based deformable models suffer from the difficulty of determining the initial contour, tuning the parameters and leakage in ill-defined edges.

In this paper, a new method of automatic brain tumor segmentation technique is proposed based on neutrosophic Kmeans clustering method. First, the non local neutrosophic wiener filtering is applied to increase the quality of the image. Then fuzzy image enhancement technique is used to enhance the filtered image. Then finally $k$ - means clustering method is used for brain tumor segmentation.

\section{Proposed Method}

\section{A. Preprocessing Using Neutrosophic Set}

Neutrosophy, a branch of philosophy introduced by Smarandache, as a generalization of dialectics, studies the origin, nature and scope of neutralities, as well as their interactions with different ideational spectra. In neutrosophy theory, every event has not only a certain degree of the truth, but also a falsity degree and an indeterminacy degree that have to be considered independently from each other [13]. Thus, a theory, event, concept, or entity, $\{S\}$ is considered with its opposite $\{A n t i-S\}$ and the neutrality $\{N e u t-S\} .\{N e u t-S\}$ is neither $\{S\}$ nor $\{A n t i-S\}$. The $\{N e u t-S\}$ and $\{A n t i-S\}$ are referred to as $\{N o n-S\}$. According to this theory, every idea $\{A\}$ is neutralized and balanced by $\{A n t i-S\}$ and
$\{$ Non $-S\}$ [13]. NS provides a powerful tool to deal with indeterminacy.

\section{B. Non local Mean Algorithm}

The non local means (NLM) algorithm is applied to the noisy MRI in order to create the reference image. In the nonlocal means [14], given a discrete noisy image $u=\{u(i) \mid i \in I\}$, the estimated value $N L[u](i)$, for a pixel $i$, is computed as a weighted average of all the pixels in the image,

$$
N L[u](i)=\sum_{j \in I} w(i, j) u(j)
$$

where the family of weights $\{w(i, j)\}_{j}$ depend on the similarity between the pixels $\mathrm{i}$ and $\mathrm{j}$, and satisfy the usual conditions $0 \leq w(i, j) \leq 1$ and $\sum_{j} w(i, j)=1$.

The similarity between two pixels $i$ and $j$ depends on the similarity of the intensity gray level vectors $u\left(N_{i}\right)$ and $u\left(N_{j}\right)$, where $N_{k}$ denotes a square neighborhood of fixed size and centered at a pixel $k$. This similarity is measured as a decreasing function of the weighted Euclidean distance, $\left\|u\left(N_{i}\right)-u\left(N_{j}\right)\right\|_{2, a}^{2}$, where $a>0$ is the standard deviation of the Gaussian kernel. The application of the Euclidean distance to the noisy neighborhoods raises the following equality

$$
E\left\|u\left(N_{i}\right)-u\left(N_{j}\right)\right\|_{2, a}^{2}=\left\|u\left(N_{i}\right)-u\left(N_{j}\right)\right\|_{2, a}^{2}+2 a^{2}
$$

This equality shows the robustness of the NLM algorithm since in expectation the Euclidean distance conserves the order of similarity between pixels.

The pixels with a similar grey level neighborhood to $u\left(N_{i}\right)$ have larger weights in the average. These weights are defined as,

$w(i, j)=\frac{1}{Z(i)} e^{-\frac{\left\|u\left(N_{i}\right)-u\left(N_{j}\right)\right\|_{2, a}^{2}}{h^{2}}}$

where $Z(i)$ is the normalizing constant

$Z(i)=\sum_{j} e^{-\frac{\left\|u\left(N_{i}\right)-u\left(N_{j}\right)\right\|_{2, a}^{2}}{h^{2}}}$

The parameter $h$ acts as a degree of filtering. It controls the decay of the exponential function and therefore the decay of the weights as a function of the Euclidean distances.

\section{The image in neutrosophic set}

The NLM filtered image is transformed into NS domain. A neutrosophic set image $P_{N S}$ is characterized by three membership sets $T, I, F$. A pixel $P$ in the image is described 
as $P(T, I, F)$ and belongs to $W$ in the following way: It is $t$ true in the set, $i$ indeterminate in the set, and $f$ false in the set, where $t$ varies in $T, i$ varies in $I$ and $f$ varies in $F$. Then the pixel $P(i, j)$ in the image domain is transformed into the neutrosophic set domain $P_{N S}(i, j)=\{T(i, j), I(i, j), F(i, j)\} . T(i, j), I(i, j)$ and $F(i, j)$ are the probabilities belong to white pixels set, indeterminate set and non white pixels set respectively [15], which are defined as:

$$
\begin{aligned}
& T(i, j)=\frac{\bar{g}(i, j)-\bar{g}_{\min }}{\bar{g}_{\max }-\bar{g}_{\min }} \\
& \bar{g}(i, j)=\frac{1}{w \times w} \sum_{m=i-w / 2}^{i+w / 2} \sum_{n=j-w / 2}^{j+w / 2} g(m, n) \\
& I(i, j)=\frac{\delta(i, j)-\delta_{\min }}{\delta_{\max }-\delta_{\min }} \\
& \delta(i, j)=a b s(g(i, j)-\bar{g}(i, j)) \\
& F(i, j)=1-T(i, j)
\end{aligned}
$$

where $\bar{g}(i, j)$ is the local mean value of the pixels of the window. $\delta(i, j)$ is the absolute value of difference between intensity $g(i, j)$ and its local mean value $\bar{g}(i, j)$.

\section{Neutrosophic image entropy}

For an image, the entropy is utilized to evaluate the distribution of the gray levels. If the entropy is the maximum, the intensities might have equal probability. If the entropy is small, the intensity distribution is non-uniform.

Neutrosophic entropy of an image is defined as the summation of the entropies of three subsets $T, I$ and $F$ [15]:

$$
\begin{aligned}
& E n_{N S}=E n_{T}+E n_{I}+E n_{F} \\
& E n_{T}=-\sum_{a=\min \{T\}}^{\max \{T\}} p_{T}(a) \ln p_{T}(a) \\
& E n_{I}=-\sum_{a=\min \{I\}}^{\max \{I\}} p_{I}(a) \ln p_{I}(a) \\
& E n_{F}=-\sum_{a=\min \{F\}}^{\max \{F\}} p_{F}(a) \ln p_{F}(a)
\end{aligned}
$$

where $E n_{T}, E n_{I}$ and $E n_{F}$ are the entropies of sets $T, I$ and $F$ respectively. $p_{T}(a), p_{I}(a)$ and $p_{F}(a)$ are the probabilities of elements in $T, I$ and $F$ respectively.

\section{E. $\omega$-wiener filtering operation}

The values of $I(i, j)$ is employed to measure the indeterminate degree of element $P_{N S}(i, j)$. The set $I$ correlated with $T$ and $F$, and the changes in $T$ and $F$ influence the distribution of element in $I$ and the entropy of $I$. A $\omega$ - wiener filtering operation for $P_{N S}, \hat{P}_{N S}(\omega)$, is defined as [15]:

$$
\begin{aligned}
& \hat{P}_{N S}(\omega)=P(\hat{T}(\omega), \hat{I}(\omega), \hat{F}(\omega)) \\
& \hat{T}(\omega)=\left\{\begin{array}{cc}
T & I<\omega \\
\hat{T}_{\omega} & I \geq \omega
\end{array}\right. \\
& \hat{T}_{\omega}(i, j)=\underset{(m, n) \in S_{i, j}}{\text { wiener }}\{T(m, n)\} \\
& \hat{F}(\omega)=\left\{\begin{array}{cc}
F & I<\omega \\
\hat{F}_{\omega} & I \geq \omega
\end{array}\right. \\
& \hat{F}_{\omega}(i, j)=\underset{(m, n) \in S_{i, j}}{\text { wiener }}\{F(m, n)\} \\
& \hat{I}_{\omega}(i, j)=\frac{\delta_{\hat{T}}(i, j)-\delta_{\hat{T} \min }}{\delta_{\hat{T} \max }-\delta_{\hat{T} \min }} \\
& \delta_{\hat{T}}(i, j)=\operatorname{abs}(\hat{T}(i, j)-\overline{\hat{T}}(i, j)) \\
& \overline{\hat{T}}(i, j)=\frac{1}{w \times w} \sum_{m=i-w / 2}^{i+w / 2} \sum_{n=j-w / 2}^{j+w / 2} \hat{T}(m, n)
\end{aligned}
$$

where $\delta_{\hat{T}}(i, j)$ is the absolute value of difference between intensity $\hat{T}(i, j)$ and its local mean value $\overline{\hat{T}}(i, j)$ at $(i, j)$ after $\omega$ - wiener filtering operation.

\section{F. Image enhancement}

Image enhancement is a basic method to improve the interpretability or perception of information in images for human viewers and it can provide better input for other automated image processing system. Here Fuzzy image enhancement technique is used for enhancing the tumor in MRI which is based on gray level mapping into fuzzy plane, using a membership transform function. The aim is to generate an image of higher contrast than the original one by giving the larger weight to the gray levels that are closer to the mean gray level of the image than to those that are farther from the mean. The intensification operation for the membership function is defined as [16],

$\mu^{\prime}(i, j)= \begin{cases}2 \mu^{2}(i, j) & \mu(i, j) \leq 0.5 \\ 1-2(1-\mu(i, j))^{2} & \mu(i, j)>0.5\end{cases}$ 


\section{G. Segmentation}

For brain tumor segmentation, $k$-means clustering technique is used [17]. Clustering is the process of partitioning or grouping a given set of patterns into disjoint clusters. This is done such that patterns in the same cluster are similar and patterns belonging to two different clusters are different. For $k$-means clustering, the number of clusters $k$ assumed to be fixed. Let the $k$ prototypes $\left(w_{1}, w_{2}, \ldots . w_{k}\right)$ be initialized to one of the $n$ input patterns $\left(x_{1}, x_{2}, \ldots . x_{n}\right)$. Therefore, $w_{l}=x_{m}, l \in\{1,2, \ldots k\}, m \in\{1,2, \ldots n\} . \quad C_{l}$ is the $l^{\text {th }}$ cluster whose value is a disjoint subset of input patterns. The quality of the clustering is determined by the following error function.

$$
E=\sum_{l=1}^{k} \sum_{x_{m} \in C_{l}}\left|x_{m}-w_{l}\right|^{2}
$$

\section{Results and Discussion}

\section{A. Materials}

The experimental datasets consist of 20 brain tumor patients MR images of T1 weighted and T2 weighted with different MRI pulse sequences. These images are obtained from PSG Institute of Medical Sciences and Research (PSG IMS \& R), Coimbatore, Tamilnadu, India. Expert's manual delineation of tumor in each MR image is treated as the ground truth. The method was implemented using MATLAB 2010a (The Math Works, Inc) on the workstation with Pentium Dual core $2.4 \mathrm{GHz}$ with 4GB of RAM and Windows XP 64-bit operating system. The segmentation results of the proposed method are given in Figure 1.

\section{B. Validation Strategies}

Several metrics are used for evaluation of the segmentations of same structure in an image [18]. Let $X$ be the set of all voxels in the image. The ground truth is defined as $T \in X$, the set of voxels that were labeled as tumor by the expert. Similarly, $S \in X$ as the set of voxels that were labeled as tumor by the proposed algorithm. The true positive set is defined as $T P=T \cap S$, i.e., the set of voxels common to $T$ and $S$. The true negative is defined as $T N=\bar{T} \cap \bar{S}$, i.e., the set of voxels that were labeled as non-tumor in both sets. The false positive set is defined as $F P=\bar{T} \cap S$, the non-tumor voxels are labeled as tumor voxels. The false negative set is defined as $F N=T \cap \bar{S}$, the tumor voxels are labeled as non-tumor voxels. From these sets, success and error rates can be computed as

Sensitivity $=\frac{|T P|}{|T P|+|F N|}$
Specificity $=\frac{|T N|}{|T N|+|F P|}$

False positive rate $(\mathrm{FNR})=\frac{|F P|}{|T N|+|F P|}$

$=1$-Specificity

False negative rate $(\mathrm{FPR})=\frac{|F N|}{|T P|+|F N|}$

$=1$-Sensitivity

Jaccard similarity metric

$J(T, S)=\frac{|T P|}{|T P|+|F P|+|F N|}$

Dice coefficient

$D(T, S)=\frac{|T P|}{\frac{1}{2}(|T P|+|F N|+|T P|+|F P|)}$

\section{Results}

The detailed experimental results of Automatic Brain tumor segmentation of the proposed method is shown in Fig. 1. The Fig. 1(a) is the Original image and the filtered image using non local NS wiener filter is shown in Fig. 1(b). The enhanced image using Fuzzy intensification, Tumor traced by experts, and the Tumor segmented by the proposed method are shown in Fig. 1(c), (d) and (e) respectively.

The evaluation result of the automatic segmentation of the proposed method is given in Table 1 .

\section{Conclusions}

In this paper, a fully automatic brain tumor segmentation technique is proposed based on neutrosophic preprocessing method and $k$-means clustering method. First, the non local neutrosophic wiener filtering is applied to increase the quality of the image. Then fuzzy image enhancement technique is used to enhance the filtered image. Then finally $k$ - means clustering method is used for brain tumor segmentation. The preliminary results of the proposed show that the $100 \%$ detection rate in all 20 cases with average of high accuracy $98.37 \%$, high specificity $99.52 \%$ and lower missing rate 0.52 . 

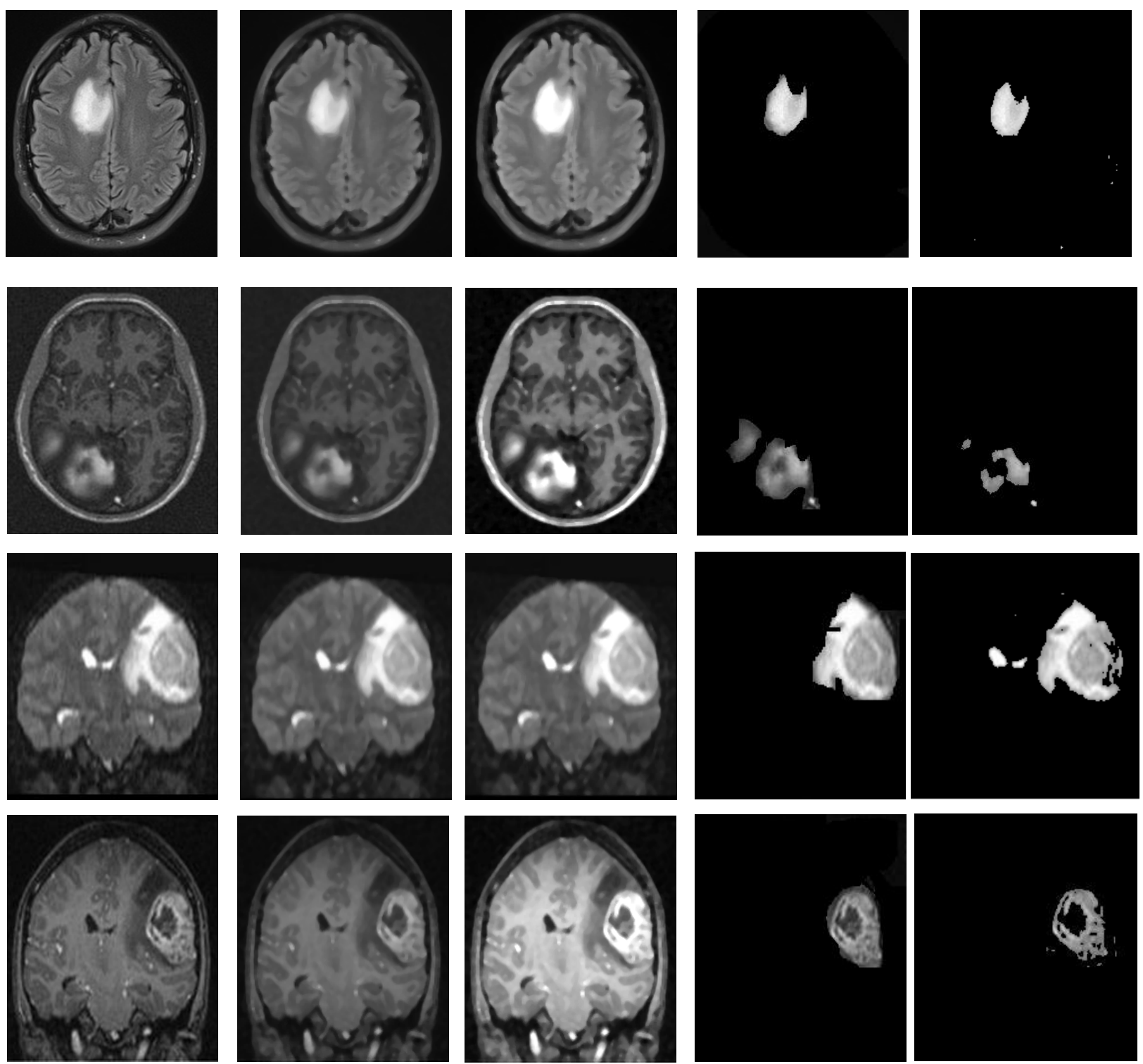

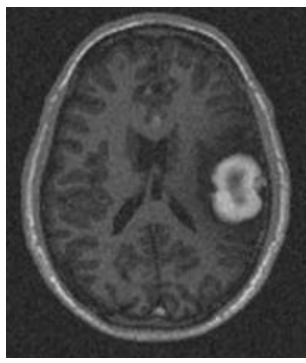

(a)

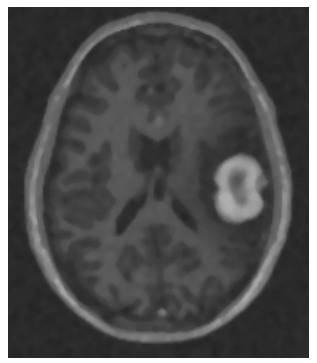

(b)

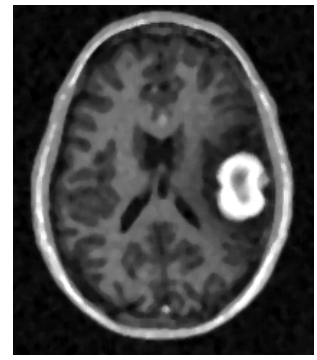

(c)

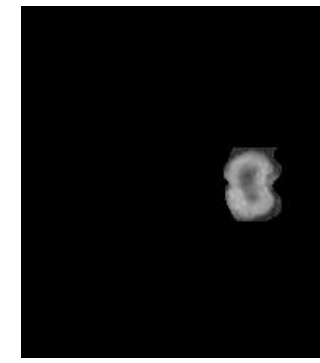

(d)

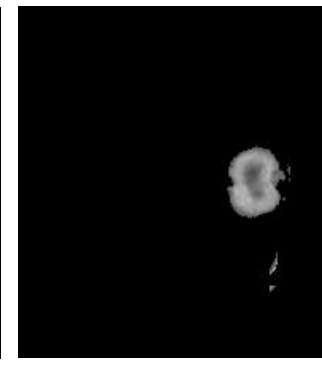

(e)

Figure 1. Results of Automatic Brain tumor segmentation of the proposed method. (a) Original image, (b) Filtered image using non local NS wiener filter, (c) Enhanced image using Fuzzy intensification (d) Tumor traced by experts, (e) Tumor segmented by the proposed method.

\section{References}

[1] Clarke L.P, Velthuizen R.P, Heine et al. MRI Segmentation: Methods and Applications. Magnetic Resonance Imaging 1995;13(3):343-368.
[2] Clark M.C, Hall L.O, Goldgof D.B, Velthuzen R, Murtagh F.R and Silbiger M.S. Automatic tumor segmentation using knowledge based techniques. IEEE Trans. On Medical Imaging 1998;17(2):187-201.

[3] Kaus M, Warfield S.K et al. Automated segmentation of MRI of brain tumors. Radiology 2001;218(2):586-591. 
TABLE I

Results of Automatic Brain Tumor Segmentation of the Proposed Method

\begin{tabular}{cccccccc}
\hline Image & Accuracy & Sensitivity & Specificity & FPR & FNR & Jaccard & Dice \\
\hline Case 1 & 0.9961 & 0.8419 & 0.9992 & 0.0008 & 0.1581 & 0.8103 & 0.8952 \\
Case 2 & 0.9681 & 0.8659 & 0.9982 & 0.0018 & 0.1341 & 0.7367 & 0.8484 \\
Case 3 & 0.9816 & 0.9054 & 0.9882 & 0.0118 & 0.0946 & 0.7960 & 0.8864 \\
Case 4 & 0.9874 & 0.8744 & 0.9935 & 0.0065 & 0.1256 & 0.8023 & 0.8703 \\
Case 5 & 0.9993 & 0.9757 & 0.9996 & 0.0004 & 0.0233 & 0.9431 & 0.9707 \\
Case 6 & 0.9861 & 0.8539 & 0.9892 & 0.0108 & 0.1461 & 0.8215 & 0.8852 \\
Case 7 & 0.9542 & 0.8734 & 0.9854 & 0.0146 & 0.1266 & 0.8365 & 0.8885 \\
Case 8 & 0.9819 & 0.9154 & 0.9982 & 0.0018 & 0.0846 & 0.8960 & 0.8964 \\
Case 9 & 0.9878 & 0.8774 & 0.9945 & 0.0045 & 0.1226 & 0.8123 & 0.8803 \\
Case 10 & 0.9953 & 0.9757 & 0.9986 & 0.0014 & 0.0233 & 0.9331 & 0.9207 \\
Case 11 & 0.9931 & 0.8615 & 0.9965 & 0.0035 & 0.1385 & 0.8726 & 0.8957 \\
Case 12 & 0.9784 & 0.9659 & 0.9982 & 0.0018 & 0.0341 & 0.8367 & 0.8788 \\
Case 13 & 0.9719 & 0.9154 & 0.9982 & 0.0018 & 0.0846 & 0.8965 & 0.8990 \\
Case 14 & 0.9943 & 0.9757 & 0.9996 & 0.0004 & 0.0233 & 0.9523 & 0.9776 \\
Case 15 & 0.9681 & 0.8672 & 0.9962 & 0.0038 & 0.1328 & 0.7367 & 0.8484 \\
Case 16 & 0.9961 & 0.8419 & 0.9992 & 0.0008 & 0.1581 & 0.8103 & 0.8952 \\
Case 17 & 0.9642 & 0.8734 & 0.9864 & 0.0136 & 0.1266 & 0.8567 & 0.8975 \\
Case 18 & 0.9876 & 0.9154 & 0.9870 & 0.0130 & 0.0846 & 0.7960 & 0.8864 \\
Case 19 & 0.9874 & 0.8744 & 0.9935 & 0.0065 & 0.1256 & 0.8023 & 0.8703 \\
Case 20 & 0.9956 & 0.9754 & 0.9943 & 0.0057 & 0.0236 & 0.9435 & 0.9710 \\
\hline Average & $\mathbf{0 . 9 8 3 7}$ & $\mathbf{0 . 9 0 1 2}$ & $\mathbf{0 . 9 9 2 6}$ & $\mathbf{0 . 0 0 5 2}$ & $\mathbf{0 . 0 9 8 5}$ & $\mathbf{0 . 8 4 4 6}$ & $\mathbf{0 . 8 9 8 1}$ \\
\hline & & & & & & & \\
\hline
\end{tabular}

[4] Moon N, Bullitt E, Leemput K.V and Gerig G. Model based brain and tumor segmentation. Proceedings in ICPR, Quebec, August, 2002: 528531.

[5] Prastawa M, Bullitt E, Ho. S and Gerig G. A brain tumor segmentation framework based on outlier detection. Med. Image Anal. 2004;8(3):275-283.

[6] Phillips W.E, Velthuizen R.P, Phuphanich S, Hall L.O, Clark M.C and Silbiger M.S. Application of fuzzy c-means segmentation technique for tissue differentiation in $\mathrm{Mr}$ images of hemorrhagic giloblastoma multiframe. Magnetic Resonance Imaging 1995;13(2):277-290.

[7] Liu J, Udupa J.K, Odhner D, Hackney D. B and Moonis G. A system for brain tumor volume estimation via MR imaging and fuzzy connectedness. Computerized Medical Imaging and Graphics 2005;29(1):21-34.

[8] Moonis G, Liu J, Udupa J.K and Hackney D. B. Estimation of tumor volume with fuzzy connectedness segmentation of MR images. American Journal of Neuroradiology 2002;23:352-363.

[9] Dou W, Ruan S, Chen Y, Bloyet D and Constants J.M. A framework of fuzzy information fusion for segmentation of brain tumor tissues on MR images. Image and Vision Computing 2007;25:164-171.

[10] Harati V, Khayati R and Farzan A. Fully automated tumor segmentation based on improved fuzzy connectedness algorithm in brain MR images. Computers in Medicine and Biology 2011;41(7):483-492.

[11] Lefohn A, Cates J, and Whitaker R. Interactive, GPU-based level sets for 3D brain tumor segmentation. MICCAI, 2003.

[12] Ho S, Bullitt E and Gerig G. Level set evolution with region competition: Automatic 3-D segmentation of brain tumors. in Proceedings ICPR, 2002:532-535.
[13] Smarandache F. A unifying field in logics Neutrosophic logic, in Neutrosophy. Neutrosophic Set, Neutrosophic Probability. third ed, American Research Press, 2003.

[14] Buades A, Coll B, and Morel J. M. A review of image denoising algorithms, with a new one. Multiscale Modeling and Simulation 2005;4:490-530

[15] Mohan, J, Krishnaveni, V and Guo Y, MRI denoising using non local neutrosophic set approach of Wiener filtering, Elsevier Biomedical Signal Processing and Control, 2013, 8(6):779-791.

[16] Pal S.K and King R.A. Image enhancement using fuzzy set. Electronics Letter 1980;16(10): 376-378.

[17] MacQueen J. Some methods for classification and analysis of multivariate observations. Proceedings of the fifth Berkeley symposium on Mathematics, Statistics and Probablity 1967:281-297.

[18] Shattuck D.W, Prasad G, Mirza M, Narr K.L and Toga A.W. Online resource for validation of brain segmentation methods. Neuroimage 2009; 45: 431-439. 